PACIFIC JOURNAL OF MATHEMATICS

Vol. 185, No. 2, 1998

\title{
PEAK SETS IN REAL-ANALYTIC CONVEX BOUNDARIES
}

\author{
RACHID BELHACHEMI
}

Suppose $D \subset \subset \mathbb{C}^{n}$ is a convex domain with real-analytic boundary. Assume $K$ is a compact subset of $\partial D$ which is a peak set for $A^{\infty}(D)$, and $L$ is a compact subset of $K$. Then $L$ is a peak set for $A^{\infty}(D)$.

\section{Introduction.}

Throughtout this paper we will denote by $D$ a smoothly bounded domain in $\mathbb{C}^{n}$ with defining function $r$.

We denote by $A^{\infty}(D)$ the set of holomorphic functions in $D$ which have $C^{\infty}$-extension to $\bar{D}$.

First, we give the necessary definitions and notations needed in this paper.

A closed subset $K \subset \partial D$ is a peak set for $A^{\infty}(D)$ if there exists a function $f \in A^{\infty}(D)$ so that $f=1$ on $K$ and $|f|<1$ on $\bar{D} \backslash K$. $K$ is locally a peak set for $A^{\infty}(D)$ if for each $p \in K$, there exists a neighborhood $V$ of $p$ so that $K \cap \bar{V}$ is a peak set for $A^{\infty}(D)$.

It is easy to see that a closed subset $K \subset \partial D$ is a peak subset for $A^{\infty}(D)$ if and only if there exists a function $g \in A^{\infty}(D)$ such that $g=0$ on $K$ and $\operatorname{Re} g>0$ on $\bar{D} \backslash K$. Such a function $g$ is called a strong support function for $K$.

We denote by $T_{p}(M)$ the real tangent space to a smooth manifold $M$ at the point $p \in M$. For a point $p \in M$, the complex tangent space of $M$ at $p$ denoted by $T_{p}^{\mathbb{C}}(M)$ is the maximal complex subspace of $T_{p}(M)$, of complex dimension $n-1$ if $M=\partial D$.

A $C^{\infty}$-submanifold $M \subseteq \partial D$ is integral at $p \in M$ if $T_{p}(M) \subseteq T_{p}^{\mathbb{C}}(\partial D) . M$ is an integral manifold if it is integral at each point $p \in M$.

A $C^{\infty}$-submanifold $M \subset \partial D$ is totally real if $T_{p}^{\mathbb{C}}(M)=\{0\}$ for every $p \in M$.

We denote by $w(\partial D)$ the set of weakly pseudoconvex boundary points.

For $p \in \partial D$, we let $N_{p}$ denote the null space in $T_{p}^{\mathbb{C}}(\partial D)$ of the Levi form at p. $D^{\alpha}$ will denote the differential operator $\frac{\partial^{|\alpha|}}{\partial x_{1}^{\alpha_{1}} \partial x_{2}^{\alpha_{2}} \cdots \partial x_{N}^{\alpha_{N}}}$ in $\mathbb{R}^{N}$, where $\alpha=\left(\alpha_{1}, \ldots, \alpha_{N}\right)$ and $|\alpha|=\alpha_{1}+\cdots+\alpha_{N}$.

We will denote by $d(z, M)$ the Euclidean distance from $z$ to a manifold M. 
The main result of this paper is:

(Theorem 1.8) $\quad$ Suppose $D \subset \subset \mathbb{C}^{n}$ is a convex domain with real-analytic boundary. Assume $K$ is a peak set for $A^{\infty}(D)$, and $L$ is a compact subset of $K$. Then $L$ is a peak set for $A^{\infty}(D)$.

Theorem 1.8 was proved by Chaumat and Chollet in [4] for strongly pseudoconvex domains with smooth boundaries in $\mathbb{C}^{n}$. Noell in [7] extended this result to convex domains with real-analytic boundaries in $\mathbb{C}^{2}$, he also showed in [7] that compact subsets of peak sets for $A^{\infty}(D)$ are peak sets for $A^{\infty}(D)$ for smooth pseudoconvex domains of finite type in $\mathbb{C}^{2}$. Furthermore, he showed that the finite type requirement can not be dropped, in fact, Noell gave in [7] an example of a pseudoconvex domain $D \subset \subset \mathbb{C}^{2}$ not of finite type, a compact subset $K$ which is a peak set for $A^{\infty}(D)$, and a compact subset $L$ of $K$ that is not a peak set for $A^{\infty}(D)$.

We recall from [2] the following useful theorem.

Theorem 1.1. Suppose $D \subset \subset \mathbb{C}^{n}$ is a convex domain with real-analytic boundary. Then for each $p \in w(\partial D)$, there exists a neighborhood $U$ of $p$ so that:

(a) $\quad w(\partial D) \cap U=\bigcup_{j=0}^{2 n-3} S_{j}$, where each $S_{j}$ is a finite disjoint union of $j$ dimensional real-analytic $C R$ submanifolds of $\partial D \cap U$. Furthermore, for all $q \in S_{j}, T_{q}\left(S_{j}\right) \cap N_{q}=\{0\}$.

(b) If $S$ is a component of some $S_{j}$ and $T_{q}(S) \subset T_{q}^{\mathbb{C}}(\partial D)$ for some $q \in S$, then $S$ is an integral submanifold of $\partial D \cap U$.

(c) $S_{j}$ is closed in $\partial D \backslash\left(\bigcup_{i=0}^{j-1} S_{i}\right), j=1, \ldots, 2 n-3$.

Theorem 1.1 shows that, analysis on convex domains with real-analytic boundaries in $\mathbb{C}^{n}$ is similar to that of a strongly pseudoconvex domain.

\section{Peak sets and integral manifolds.}

The purpose of this section is to build additional ingredients that will enable us to prove the main result of this paper Theorem 1.8. In this section we will resolve two questions, in the first result (Theorem 1.2) we will show that the intersection of a peak set and any strata of $w(\partial D)$ described in Theorem 1.1 is locally contained in integral manifolds. The second result (Theorem 1.4) contains our estimate which shows the local behavior of strong support functions in convex domains with real-analytic boundaries.

Theorem 1.2. Suppose $D \subset \subset \mathbb{C}^{n}$ is a convex domain with real-analytic boundary. Assume $K$ is a compact subset of $\partial D$ which is locally a peak set

\footnotetext{
${ }^{1}$ I would like to thank my advisor, Dr. Alan Noell for his useful suggestions. His encouragement and guidance have been invaluable to me.
} 
for $A^{\infty}(D)$. Let $S$ be any strata of $w(\partial D)$ as in Theorem 1.1, and suppose $\operatorname{dim}_{\mathbf{R}} S=2 t+\lambda$, where $\operatorname{dim}_{\mathbb{C}} T_{q_{0}}^{\mathbb{C}}(S)=t$ for all $q_{0} \in S$. Suppose $p \in K \cap S$. Then there exists a neighborhood $U \subset \mathbb{C}^{n}$ of $p$, a holomorphic change of coordinates in $U$, in which $p=0$ and $S \subset \mathbb{C}^{t+\lambda} \times\{0\}$, a neighborhood $U^{\prime} \subset$ $\mathbb{C}^{t+\lambda}$ of 0 , a strongly pseudoconvex domain $\Omega \subset \subset U^{\prime}$, a locally peak set $\widetilde{L} \subset \partial \Omega \cap V^{\prime}$, where $V^{\prime} \subset \subset U^{\prime}$ is a neighborhood of zero, and a totally real smooth $\widetilde{M} \subset \partial \Omega \cap V^{\prime}$ so that:

(a) $K \cap S \cap V \subseteq \widehat{L} \subset \widehat{M} \subset(\partial \Omega \times\{0\}) \cap V^{\prime \prime} \subset \partial D \cap V$, where $\widehat{L}=\widetilde{L} \times\{0\}$, $\widehat{M}=\widetilde{M} \times\{0\}, V^{\prime \prime}=V^{\prime} \times\{0\}$, and $V \subset \subset U$ is a neighborhood of 0.

(b) $T_{q}(\widehat{M}) \subseteq T_{q}^{\mathbb{C}}(\partial D)$ for all $q \in \widehat{M}$.

(c) $\operatorname{dim}_{\mathbf{R}} \widehat{M} \leq n-2$.

Proof.

Case 1. Assume that $S$ is not integral at $p$.

Since $S$ is a real-analytic CR manifold, then by Rossi's theorem [9] there exists a neighborhood $U$ of $p$ in $\mathbb{C}^{n}$ and a biholomorphic map $\Phi: U \rightarrow \mathbb{C}^{n}$ so that $\Phi(p)=0$ and $\Phi(U \cap S) \subset \mathbb{C}^{t+\lambda} \times\{0\}$. Note that Rossi's theorem enables us to put real-analytic CR manifolds of $\mathbb{C}^{n}$ into lower dimensional $\mathbb{C}^{k}(k<n)$.

Let $z \in U$, and $\Phi(z)=\left(z^{\prime}, z^{\prime \prime}\right)$, with $z^{\prime}=\left(z_{1}, \ldots, z_{t+\lambda}\right)$, denotes the new holomorphic change of coordinates near 0 , where $z_{t+\lambda}=u+i v$ is the complex normal direction to $\partial D$ at 0 . We assume that the new manifold obtained under $\Phi$ that sits in $\mathbb{C}^{t+\lambda} \times\{0\}$ is also denoted by $S$.

We define the function $\rho$ as follows:

$$
\rho\left(z^{\prime}\right)=r \circ h\left(z^{\prime}\right)
$$

where $h\left(z^{\prime}\right)=\left(z^{\prime}, 0, \ldots, 0\right)$.

Let $U^{\prime}$ be a neighborhood of $0^{\prime}$ in $\mathbb{C}^{t+\lambda}$, and put

$$
\Omega=\left\{z^{\prime} \in U^{\prime}: \rho\left(z^{\prime}\right)<0\right\}
$$

Note that $\Omega$ is a bounded domain in $U^{\prime}$, and $S$ is locally contained in $(\partial \Omega \times$ $\{0\}) \cap U^{\prime}$, with $\partial \Omega \cap U^{\prime}=\left\{z^{\prime} \in U^{\prime}: \rho\left(z^{\prime}\right)=0\right\}$.

We need to show that $\rho$ is a defining function for $\Omega$, so it suffices to show that if $U^{\prime}$ is small enough, then $\nabla \rho \neq 0$ on $\partial \Omega \cap U^{\prime}$. 
Assume $\frac{\partial r}{\partial z_{t+\lambda}}(0)=1$. By the chain Rule we have,

$$
\begin{aligned}
\frac{\partial \rho}{\partial z_{t+\lambda}}\left(0^{\prime}\right) & =\sum_{k=1}^{n} \frac{\partial r}{\partial w_{k}}(0) \cdot \frac{\partial h_{k}}{\partial z_{t+\lambda}}\left(0^{\prime}\right)+\sum_{k=1}^{n} \frac{\partial r}{\partial \bar{w}_{k}}(0) \cdot \frac{\partial \bar{h}_{k}}{\partial z_{t+\lambda}}\left(0^{\prime}\right) \\
& =\sum_{k=1}^{n} \frac{\partial r}{\partial w_{k}}(0) \cdot \frac{\partial h_{k}}{\partial z_{t+\lambda}}\left(0^{\prime}\right)=1
\end{aligned}
$$

Thus $\nabla \rho\left(0^{\prime}\right) \neq 0$, and hence $\nabla \rho \neq 0$ in a neighborhood of $0^{\prime}$.

To show that $\Omega$ is strongly pseudoconvex near $0^{\prime}$, it is enough to show that $\Omega$ is strongly pseudoconvex at $0^{\prime}$. An easy computation of the Levi form yields,

$$
\begin{aligned}
L_{\rho}\left(0^{\prime}, \eta\right) & =L_{r}\left(0, h^{\prime}\left(0^{\prime}\right) \eta\right) \\
& =L_{r}\left(0,\left(\eta_{1}, \ldots, \eta_{t+\lambda}, 0, \ldots, 0\right)\right) .
\end{aligned}
$$

(Here $L_{r}(r, p)$ denotes the Levi form of $r$ at $\left.p.\right) \eta=\left(\eta_{1}, \ldots, \eta_{t+\lambda}\right) \in \mathbb{C}^{t+\lambda}$, $\eta \neq 0^{\prime}$ and $\eta \in T_{0^{\prime}}^{\mathbb{C}}(\partial \Omega)$. Since $T_{0}(S) \cap N_{0}=\{0\}$ by Theorem 1.1, we may conclude that $L_{\rho}\left(0^{\prime}, \eta\right)>0$.

Now, we show that there exists locally a peak set $\widetilde{L} \subset \partial \Omega$ for $A^{\infty}(\Omega)$ so that

$$
K \cap S \cap V \subseteq \widetilde{L} \times\{0\}
$$

where $V \subset \subset U$ is a neighborhood of 0 in $\mathbb{C}^{n}$.

Let $f$ be a strong support function for $K \cap \bar{V}$. Define the function $g$ as follows,

$$
g\left(z^{\prime}\right)=f \circ h\left(z^{\prime}\right)
$$

and

$$
\widetilde{L}=\left\{z^{\prime} \in \bar{\Omega} \cap V^{\prime}: g\left(z^{\prime}\right)=0\right\},
$$

with $V^{\prime} \subset U^{\prime}$ a neighborhood of $0^{\prime}$.

First we claim that $g$ is a strong support function for $\widetilde{L} \cap V^{\prime}$. It is obvious that $g \in A^{\infty}(\Omega)$. Now let us show that $g \not \equiv 0$ on $\bar{\Omega} \cap V^{\prime}$. Assume to the contrary that $g \equiv 0$ on $\bar{\Omega} \cap V^{\prime}$. Then by the Chain Rule we get,

$$
0=\frac{\partial g}{\partial z_{t+\lambda}}\left(0^{\prime}\right)=\sum_{k=1}^{n} \frac{\partial f}{\partial z_{k}}(0) \cdot \frac{\partial h_{k}}{\partial z_{t+\lambda}}\left(0^{\prime}\right)=\frac{\partial f}{\partial z_{t+\lambda}},
$$

and so $\frac{\partial f}{\partial u}(0)=0$. But this is absurd because Re $f$ is a non-constant pluriharmonic function on $D$ which has a local minimum at 0 , and therefore by Hopf lemma, we must have $\frac{\partial(\operatorname{Re} f)}{\partial u}(0)<0$. 
We note that if $\operatorname{Re} f=0$ then $g=0$, and hence $\operatorname{Re} g>0$ on $\bar{\Omega} \backslash \widetilde{L} \cap V^{\prime}$, moreover, $\widetilde{L} \subset \partial \Omega \cap V^{\prime}$ by the maximum modulus principle.

Let $z \in K \cap S \cap V$ with $z=\left(z^{\prime}, 0, \ldots, 0\right)$. Then $g\left(z^{\prime}\right)=f \circ h\left(z^{\prime}\right)=$ $f\left(z^{\prime}, 0, \ldots, 0\right)=0$ since $z \in K$. So $\left(z^{\prime}, 0^{\prime}\right) \in \widetilde{L} \times\{0\}$, and this gives (1).

Now, we verify properties (a)-(c) of the theorem.

Since $\Omega$ is a strongly pseudoconvex domain near $0^{\prime}$, and $\widetilde{L}$ is locally a peak set for $A^{\infty}(\Omega)$, then by a result of Chaumat and Chollet in [4] (Theorem 7), we obtain a totally real integral submanifold of $\widetilde{M} \subset \partial \Omega \cap V^{\prime}$ if $V^{\prime}$ is small enough, so that $\widetilde{L} \cap V^{\prime} \subset \widetilde{M}$.

We put

$$
\widehat{M}=\widetilde{M} \times\{0\}
$$

Then, for all $q \in \widehat{M}$

$$
T_{q}(\widehat{M}) \subseteq T_{q}^{\mathbb{C}}(\partial D)
$$

and

$$
\operatorname{dim}_{\mathbf{R}} \widehat{M}=t+\lambda-1 \leq(n-1)-1=n-2 .
$$

This completes the proof in the case $T_{p}(S) \nsubseteq T_{p}^{\mathbb{C}}(\partial D)$.

Case 2. If $S$ is integral at $p$, then $S$ is an integral submanifold of $\partial D$ by Theorem 1.1 (b). Hence by Theorem 1.1 (a), and a result of Bedford and Fornaess in [1] (Lemma 3, p. 287), we must have $S$ totally real, and therefore the preceding proof of Case 1 is easily modified. This finishes the proof of Theorem 1.2.

Remark 1.3. Observe that the convexity of $D$ was used only to get a real-analytic strata described in Theorem 1.1.

First, we introduce the function $S_{R}(f)$, and then we state and prove Theorem 1.4.

For $R>0$, let $S_{R}(f)=f-R f^{2}$, where $f$ is a strong support function for a closed subset $K \subset \partial D$. We note that,

$$
\operatorname{Re}\left(S_{R}(f)\right)=\operatorname{Re} f(1-R(\operatorname{Re} f))+R(\operatorname{Im} f)^{2},
$$

and for a small neighborhood $U$ of $K$,

$$
S_{R}(f)=0 \text { on } K \quad \text { and } \operatorname{Re}\left(S_{R}(f)\right)>0 \text { on }(\bar{D} \cap U) \backslash K .
$$

Theorem 1.4. Suppose $D \subset \subset \mathbb{C}^{n}$ is a convex domain with real-analytic boundary. Let $K \subset \partial D$ be a compact subset which is a peak set for $A^{\infty}(D)$ with strong support function $f$. Let $p, S, \widetilde{L}, \widehat{L}, \Omega, U^{\prime}$ and $U$ be as in Theorem 1.2. Let $R$ be a sufficiently large positive number. Then there exist neighborhoods $V \subset \subset U$ of $p, V^{\prime} \subset \subset U^{\prime}$ of $p^{\prime}$, a totally real manifold $M^{\prime} \subset \partial \Omega \cap V^{\prime}$ of 
dimension at most $(n-1)$ containing $\widetilde{L} \cap V^{\prime}$, and a smooth manifold $N \subset V$ containing $M=M^{\prime} \times\{0\}$ so that

(a) $K \cap S \cap V \subseteq \widehat{L} \subset M \subset(\partial \Omega \times\{0\}) \cap V^{\prime \prime} \subset \partial D \cap V$, with $V^{\prime \prime}=V^{\prime} \times\{0\}$.

(b) $\operatorname{Re} S_{R}(f)(q) \geq c d^{2}(q, N)$ if $q \in \bar{D} \cap V$, for some positive constant $c$.

Proof. By Theorem 1.2, we know that $\Omega \subset \subset U^{\prime}$ is a strongly pseudoconvex domain with boundary, and $\widetilde{L}$ is locally a peak set for $A^{\infty}(\Omega)$ with strong support function $g$, so by using a result of Chaumat and Chollet in [4] (Proposition 9), we can find a neighborhood $V^{\prime} \subset \subset U^{\prime}$ of $0^{\prime}$ and a totally real submanifold $M^{\prime} \subset \partial \Omega \cap V^{\prime}$ of dimension $t+\lambda \leq n-1$, containing $\widetilde{L} \cap V^{\prime}$ such that $\operatorname{Re} g\left(q^{\prime}\right) \geq c^{\prime} d^{2}\left(q^{\prime}, M^{\prime}\right)$ for all $q^{\prime} \in \bar{\Omega} \cap V^{\prime}$, where $c$ is a positive constant. Using this and the reasoning in Theorem 1.2 (a), we get part (a) of the theorem.

Define the manifold $N \subset U$ by

$$
N=M^{\prime} \times\left\{\mathbb{C}^{n-t-\lambda}\right\},
$$

and observe that $M=M^{\prime} \times\{0\} \subset N$. It remains to show part (b).

Let $q^{\prime} \in M$. Since $M$ is totally real, we can make a holomorphic linear change of coordinates near $q^{\prime}$ that we denote by $z_{j}=x_{j}+i y_{j}, j=1, \ldots, t+$ $\lambda-1, z_{t+\lambda}=u+i v$ so that $q^{\prime}=0$, and $T_{0}\left(M^{\prime}\right)=\left\{z^{\prime} \in \mathbb{C}^{t+\lambda}: y_{1}=\right.$ $\left.\cdots=y_{t+\lambda-1}=u=0\right\}$. We may assume that $T_{0}(\partial \Omega)=\left\{z^{\prime}: u=0\right\}$ and $T_{0}(\partial D)=\left\{\left(z^{\prime}, z^{\prime \prime}\right): u=0\right\}$.

Let $g\left(z^{\prime}\right)=f\left(z^{\prime}, 0, \ldots, 0\right)$ be the strong support function for $\widetilde{L} \cap \overline{V^{\prime}}$ constructed in the proof of Theorem 1.2. The proof of Proposition 9 in [4] guarantees that the real Hessian of $\operatorname{Re} g$ at 0 is positive definite when restricted to the orthgonal complement of $T_{0^{\prime}}\left(M^{\prime}\right)$ in $T_{0^{\prime}}^{\mathbb{C}}(\partial \Omega)$. In addition, the Cauchy-Riemann equations and the Hopf lemma give $\frac{\partial(\operatorname{Re} f)}{\partial u}(0)<0$. Using this, we may conclude that for all $q \in \bar{D}$ near $q^{\prime}$, $\operatorname{Re} S_{R}(f)(q) \geq c d^{2}(q, N)$. This ends the proof of Theorem 1.4.

The proposition below is due to Harvey and Wells and appears in [5]. We will use it in the proof of Theorem 1.8.

Proposition 1.5. Suppose $\widehat{M} \subset \partial D \cap U$ is a totally real submanifold, where $U$ is an open subset of $\mathbb{C}^{n}$. Let $\chi$ be a $C^{\infty}$ function in $\widehat{M}$. Then there exists a $C^{\infty}$ function $\tilde{\chi}$ in $U$ so that:

(1) $\tilde{\chi}=\chi$ on $\widehat{M}$.

(2) $\bar{\partial} \widetilde{\chi}$ vanishes to infinite order along $\widehat{M}$, i.e. $D^{\alpha}(\bar{\partial} \widetilde{\chi}) \equiv 0$ along $\widehat{M} \cap U$ for each multi-index $\alpha$.

(3) $\tilde{\chi}$ is locally constant near where $\chi$ is locally constant.

(4) If $\chi$ has compact support in $\widehat{M}$, then $\tilde{\chi}$ has compact support in $U$. 
(5) First derivatives of $\tilde{\chi}$ vanishes on $\widehat{M}$ in directions perpendicular to $T(\widehat{M})+J T(\widehat{M})$, where $J$ is the almost complex structure corresponding to multiplication by $i$.

\section{Compact subsets of peak sets.}

The next proposition goes back to Chaumat and Chollet [3]. Proposition 1.6 allows us to construct peak functions from the functions stated there.

Proposition 1.6. Suppose $D \subset \mathbb{C}^{n}$ is a bounded pseudoconvex domain with smooth boundary. Let $E$ be a compact subset of $\partial D, W$ a neighborhood of $E$ in $\mathbf{C}^{n}$, and $\rho$ a non-negative continuous function on $W$ which vanishes on E. Suppose that there exists a function $G \in C^{\infty}(W \cap \bar{D})$ such that:

(a) $E=\{z \in W \cap \bar{D}: G(z)=0\}$.

(b) For each $\alpha \in \mathbb{N}^{n}$, and for each $k \in \mathbb{N}$ there exists $C_{\alpha k}>0$ such that for each $z \in W \cap \bar{D}$

$$
\left|D^{\alpha}(\bar{\partial} G(z))\right| \leq C_{\alpha k}[\rho(z)]^{k}
$$

(c) There exists a constant $c>0$ so that for all $z \in \bar{D} \cap W$,

$$
\operatorname{Re} G(z) \geq c \rho(z)
$$

Then $E$ is a peak set for $A^{\infty}(D)$.

Remark 1.7. Let $\left\{E_{j}\right\}_{j=1}^{\infty}$ be a collection of peak sets for $A^{\infty}(D)$, with strong support functions $\left\{f_{j}\right\}_{j=1}^{\infty}$, then $E=\cap_{j=1}^{\infty} E_{j}$ is a peak set for $A^{\infty}(D)$. To see this, let $c_{j}=\max \left\{\left\|D^{\alpha} f_{j}\right\|_{\infty}: 0 \leq|\alpha| \leq j\right\}$, and put $f=\sum_{j=1}^{\infty} \frac{1}{c_{j^{2}}} f_{j}$. We note that since $\left\|f_{j}\right\|_{\infty} \leq c_{j}$, so $\left\|\frac{1}{c_{j}} f_{j}\right\|_{\infty} \leq 1$, then $f$ is well-defined.

Moreover, $f \in A^{\infty}(D)$ and $\operatorname{Re} f_{j}(z) \geq 0$ for all $j$ and $z \in \bar{D}$, and $z \in E$ if and only if $f(z)=0$. Therefore $f$ is a strong support function for $E$.

Now, we are ready to prove our main result. The approach which we have carried out to prove Theorem 1.8 is based on that used by Chaumat and Chollet in [4].

Theorem 1.8. Suppose $D \subset \subset \mathbb{C}^{n}$ is a convex domain with real-analytic boundary. Let $K$ be a compact subset of $\partial D$ which is a peak set for $A^{\infty}(D)$, and $L$ a compact subset of $K$. Then $L$ is a peak subset for $A^{\infty}(D)$.

Proof. We apply Theorem 1.1 to get a finite covering of $K \cap w(\partial D)$ by open sets $\left\{U_{\beta}^{\prime}\right\}_{\beta=1}^{l}$ so that on each $U_{\beta}^{\prime},(1 \leq \beta \leq l)$ properties $(\mathrm{a}) \rightarrow(\mathrm{c})$ of Theorem 
1.1 are satisfied. We will take compact subsets within each $U_{\beta}^{\prime}$ and then take intersections.

Fix $\beta$, and drop the subscript from $U_{\beta}^{\prime}$.

The idea of the proof is to take compact subsets in $U^{\prime}$ successively on $S_{2 n-3}, S_{2 n-4}, \ldots, S_{0}$ where $S_{j}(0 \leq j \leq 2 n-3)$ is a real-analytic strata of $w(\partial D)$ described in Theorem 1.1 starting with the maximal dimensional strata $S_{2 n-3}$.

Let

$$
L_{1}=[K \cap w(\partial D)] \cup L .
$$

Noell in [7] proved that $L_{1}$ is a peak set for $A^{\infty}(D)$ in pseudoconvex with smooth boundary in $\mathbb{C}^{2}$, and Iordan in [6] generalized the result to such domains in $\mathbb{C}^{n}$. Observe that $L \subseteq L_{1} \subseteq K$, and one can take compact subsets of $K$ away from $w(\partial D)$.

We put

$$
L_{2}=\left[L_{1} \cap\left(S_{0} \cup S_{1} \cup \cdots S_{2 n-4}\right)\right] \cup L
$$

and note that $L \subseteq L_{2} \subseteq L_{1} \subseteq K$. We will show that $L_{2}$ is a peak set for $A^{\infty}(D)$. We will remove from $L_{1}$ points of $(K \backslash L)$ on $S_{2 n-3}$.

Let $\left\{V_{k}\right\}_{k=1}^{\infty}$ be a family of open neighborhoods of $L_{1} \cap\left(S_{0} \cup \cdots \cup S_{2 n-4}\right)$ such that $V_{k+1} \subset \subset V_{k}$ and $\cap_{k=1}^{\infty} V_{k}=L_{1} \cap\left(S_{0} \cup \cdots \cup S_{2 n-4}\right)$.

Fix $k$, and let $U$ be a neighborhood of $V_{k}$. We first show that $\left(L_{1} \cap \bar{V}_{k}\right) \cup L$ is a peak set for $A^{\infty}(D)$. Using this and Remark 1.7 we obtain that $L_{2}$ is a peak set for $A^{\infty}(D)$.

Lemma 1.9. Let $L_{1}, L, \bar{V}_{k}$ and $U$ be as above. Then there exists a peak set $L^{\prime} \subset \partial D$ for $A^{\infty}(D)$ so that:

(1) $L^{\prime} \subset L_{1}$

(2) $L^{\prime} \backslash U=L \backslash U$.

(3) $L \cup\left(L_{1} \cap \bar{V}_{k}\right) \subset L^{\prime}$.

Proof. Let $f$ be a strong support function for $L_{2}$. Apply Theorem 1.4 to get an open covering for $L_{2} \backslash U$ by open sets $U_{j}^{\prime}$ and $U_{j}$, with $U_{j}^{\prime} \subset \subset U_{j}$ $(1 \leq j \leq l)$, a smooth manifold $N_{j} \subset U_{j}^{\prime}$, and a constant $c_{j}$ such that $L_{1} \cap S_{2 n-3} \cap U_{j}^{\prime} \subset N_{j}(1 \leq j \leq l)$, and for each $z \in \bar{D} \cap U_{j}^{\prime}$

$$
\operatorname{Re} S_{R}(f)(z) \geq c_{j} d^{2}\left(z, N_{j}\right) .
$$

Let $\chi_{j}^{\prime}: \mathbb{C}^{n} \rightarrow[0,1]$ be a $C^{\infty}$-function so that $\chi_{j}^{\prime} \equiv 1$ on $U_{j}(1 \leq j \leq l)$ and $\operatorname{supp} \chi_{j}^{\prime} \subset U_{j}^{\prime}$. For $z \in \mathbb{C}^{n}$, put

$$
\rho(z)=\sum_{j=1}^{l} \chi_{j}^{\prime}(z) d^{2}\left(z, N_{j}\right) .
$$


Then $\rho \geq 0$, and $\rho \equiv 0$ on $L_{1} \cap S_{2 n-3}$. In addition, (2) gives for each $z \in \bar{D}, \sum_{j=1}^{l} \chi_{j}^{\prime}(z) \operatorname{Re} S_{R}(f)(z) \geq \sum_{j=1}^{l} c_{j} \chi_{j}^{\prime}(z) d^{2}\left(z, N_{j}\right) \geq c_{0} \rho(z)$, with $c_{0}=$ $\min \left\{c_{j}\right\}_{j=1}^{l}$. Thus

$$
\operatorname{Re} S_{R}(f)(z) \geq \frac{c_{0}}{l} \rho(z)
$$

Let $\left\{\chi_{j}\right\}_{j=1}^{l}$ be a partition of unity on $L_{1} \backslash U$ subordinate to the cover $\left\{U_{j}\right\}_{j=1}^{l}$ so that for $1 \leq j \leq l \chi_{j} \in C^{\infty}\left(U_{j}\right), \operatorname{supp} \chi_{j} \subset U_{j}$, and

$$
\sum_{j=1}^{l} \chi_{j}=1 \quad \text { on } L_{1} \text {. }
$$

Put $\Omega_{1}=\cup_{j=1}^{l} U_{j}$ and $\Omega_{2}=\Omega_{1} \cup U$. Let $D^{\prime}$ be a compact neighborhood of $D$ containing $\Omega_{2}$. Choose a $C^{\infty}$-function $s$ on $\mathbb{C}^{n}$ so that $s \geq 0$, supp $s \subset D^{\prime}$,

$$
L=\left\{z \in D^{\prime}: s(z)=0\right\}
$$

Let $M_{j}=M_{j}^{\prime} \times\{0\}(1 \leq j \leq l)$ be the totally real manifold obtained from Theorem 1.4 and which is contained in $N_{j}$. Let $z^{(j)}=\left(z^{\prime(j)}, z^{\prime \prime(j)}\right)$ be the holomorphic coordinate system on $U_{j}^{\prime}(1 \leq j \leq l)$ described in the proof of Theorem 1.2.

Put

$$
s_{j}=s \chi_{j}
$$

We apply Proposition 1.5 to $s_{j}$ restricted to $M_{j}^{\prime}$ in a neighborhood of $V_{j}^{\prime} \subset$ $\mathbb{C}^{t+\lambda}$ to get a function $\widehat{s}_{j} \in C^{\infty}\left(\mathbb{C}^{t+\lambda}\right)$ so that $\widehat{s}_{j}=s_{j}$ on $M_{j}^{\prime}$, supp $\widehat{s}_{j} \subset V_{j}^{\prime \prime}$, $V_{j}^{\prime \prime} \subset \subset V_{j}^{\prime}$, and $D^{\alpha}\left(\bar{\partial} \widehat{s}_{j}\right) \equiv 0$ on $M_{j}^{\prime}$ for each multi-index $\alpha$. Furthermore, since $\bar{\partial} \widehat{s}_{j} \equiv 0$ on $M_{j}^{\prime}$, then as a consequence of the Cauchu-Riemann equations, we get the differential of $\operatorname{Re} \widehat{s}_{j}$ is zero on $J T\left(M_{j}^{\prime}\right)$.

Extend $\widehat{s}_{j}$ trivially to get a function $\widetilde{s}_{j}$ on $\mathbb{C}^{n}$ defined by $\widetilde{s}_{j}\left(z^{(j)}\right)=\widehat{s}_{j}(z)$. Then $\widetilde{S}_{j}=s_{j}$ on $N_{j}$ (recall that $N_{j}=M_{j}^{\prime} \times \mathbb{C}^{n-t-\lambda}$ ) and $\bar{\partial} \widetilde{s}_{j}$ vanishes to infinite order along $N_{j} \cap U_{j}^{\prime}$. We modify $\widetilde{s}_{j}$ away from $M_{j}$ to get $\operatorname{supp} \widetilde{s} \subset U_{j}^{\prime}$ if $U_{j}^{\prime}$ is small enough. In addition, the differential of $\operatorname{Re} \widetilde{s}_{j}$ vanishes on $J T\left(N_{j}\right)$, this is true because $\bar{\partial} \widetilde{s}_{j}=\bar{\partial} \widehat{s}=0$.

We deduce from this, (6), and (7) by Taylor expanding $\operatorname{Re} \widetilde{s}_{j}$ that there exists a constant $c_{j}^{\prime}>0$ such that for each $z \in \mathbb{C}^{n}, \operatorname{Re} \widetilde{s}_{j}(z) \geq-c_{j}^{\prime} d^{2}(z, N)$.

Let

$$
\widetilde{s}=\sum_{j=1}^{l} \widetilde{s}_{j} .
$$


We may apply the manipulations used by Chaumat and Chollet in [4] to our situation, and deduce that there exist constants $C_{\alpha k}>0$ and $d>0$ such that for each $z \in \mathbb{C}^{n}, \alpha \in \mathbb{N}^{n}$, and $k \in \mathbb{N}$,

$$
\left|D^{\alpha} \bar{\partial} \widetilde{s}(z)\right| \leq C_{\alpha k}[\rho(z)]^{k}
$$

and

$$
\operatorname{Re} \widetilde{s}(z) \geq-d \rho(z)
$$

Define the function $G$ by

$$
G=S_{R}(f)+\delta \widetilde{s},
$$

where $\delta>0$ is sufficiently small. Using (4) and (9), there exists a positive constant $c$ so that,

$$
\operatorname{Re} G(z) \geq c \rho(z) .
$$

Let

$$
L^{\prime}=\left\{z \in \bar{D} \cap \Omega_{2}: G(z)=0\right\},
$$

and note by (6) that $L^{\prime} \subset\left\{z \in \bar{D} \cap \Omega_{2}: \rho(z)=0\right\}$.

Thus, by Proposition 1.6, we may conclude that $L^{\prime}$ is a peak set for $A^{\infty}(D)$. Now let us verify properties $(1) \rightarrow(3)$ stated in Lemma 1.9.

(1) There are two cases to consider:

(a) If $z \in \Omega_{1} \cap L_{1}^{\prime}$, then by (5) we have $\widetilde{s}(z)=\sum_{j=1}^{l} \chi_{j}(z) s(z)=s(z)$, and since $\operatorname{Re} G=0$ on $L^{\prime}$, we get $s(z)=0$, so by $(6) z \in L$, and hence $\in L_{1}$.

(b) If $z \in\left(U \backslash \Omega_{1}\right) \cap L_{1}^{\prime}$, then $\chi_{j}^{\prime}(z)=0$ for all $j(1 \leq j \leq l)$ and this gives $\widetilde{s}(z)=0$, and so $\operatorname{Re} G(z)=\operatorname{Re} S_{R}(f)(z)=0$. Thus $z \in L_{1}$. Combining the two cases we obtain $L^{\prime} \subset L_{1}$.

(2) This follows from (a) and by observing that

(c) $\Omega_{1} \cap L \subset L^{\prime}$. (If $z \in \Omega_{1} \cap L$, then $\widetilde{s}(z)=0$ and $S_{R}(f)(z)=0$, so $G(z)=0$ and this gives $z \in L^{\prime}$.)

(3) Finally, (3) follows from (c) and the following:

(d) If $z \in L_{1} \cap \bar{V}_{k}$, then $z \notin \Omega_{1}$ and so $\widetilde{s}(z)=0$. Since $S_{R}(f)(z)=0$ we have $G(z)=0$, and hence $z \in L^{\prime}$.

(e) If $z \in\left(U \backslash \Omega_{1}\right) \cap L$, then $\widetilde{s}(z)=s(z)=0$. Because $S_{R}(f)(z)=0$, we get $G(z)=0$ and hence $z \in L_{1}$. This completes the proof of Lemma 1.9.

Now we proceed with the proof that $L_{2}$ is a peak set for $A^{\infty}(D)$. Let $\left\{U_{i}\right\}_{i=1}^{\infty}$ be a family of neighborhoods of $\bar{V}_{k}$ so that $U_{i+1} \subset \subset U_{i}$ and $\cap_{i=1}^{\infty} U_{i}=$ 
$\bar{V}_{k}$. By virtue of Lemma 1.9, we get for each $i \geq 1$ a peak set $L_{i}^{\prime} \in A^{\infty}(D)$ so that properties $(1) \rightarrow(3)$ hold. Thus by Remark 1.7, $L^{\prime}=\cap_{i=1}^{\infty} L_{i}$ is a peak set for $A^{\infty}(D)$, and by part (3) of Lemma 1.9 we have $L \cup\left(L_{1} \cap \bar{V}_{k}\right) \subset L^{\prime}$. Furthermore, for each $i \geq 1, L_{1}^{\prime}=\left[L \cup\left(L_{1} \cap \bar{V}_{k}\right)\right] \cup L_{i}^{\prime \prime}$, with $L_{i}^{\prime \prime} \subset(K \cap$ $\left.U_{i}\right) \backslash\left(K \cap \bar{V}_{k}\right)$. Taking intersections, we obtain $L^{\prime}=L \cup\left(L_{1} \cap \bar{V}_{k}\right)$. Thus $L \cup\left(L_{1} \cap \bar{V}_{k}\right)$ is a peak set for $A^{\infty}(D)$, and therefore $L_{2}$ is a peak set for $A^{\infty}(D)$.

Let

$$
L_{3}=\left[L_{2} \cap\left(S_{0} \cup \cdots \cup S_{2 n-5}\right)\right] \cup L .
$$

We proceed along the same lines of the proof that $L_{2}$ is a peak set for $A^{\infty}(D)$, removing from $L_{2}$ points of $(K \backslash L)$ on $S_{2 n-4}$ to obtain that $L_{3}$ is a peak set for $A^{\infty}(D)$.

Continuing inductively and using the same process as above, we finally obtain that

$$
L_{m}=\left(L_{m-1} \cap S_{0}\right) \cup L
$$

is a peak set for $A^{\infty}(D)$, with $m=2 n-2$ and $L_{m-1}$ is a peak set for $A^{\infty}(D)$.

Now let us show that $L$ is a peak set for $A^{\infty}(D)$. We use Proposition 1.6. Choose a neighborhood $W$ of $L$ so that $W$ does not contain the points of $\left(S_{0} \cap L_{m}\right) \backslash L$, these points are isolated in $L_{m}$.

Let $h$ be a strong support function for $L_{m}$.

Put

$$
G=h \quad \text { on } W
$$

and

$$
\rho=0 \text {. }
$$

By Proposition 1.6, $L$ is a peak set for $A^{\infty}(D)$.

\section{References}

[1] E. Bedford and J.E. Fornaess, Complex manifolds in pseudoconvex boundaries, Duke Math. J., 48 (1981), 279-288.

[2] R. Belhachemi, Local versus global peak sets in real-analytic convex boundaries, reprint, 1996.

[3] J. Chaumat and A.M. Chollet, Ensembles pics pour $A^{\infty}(D)$, Ann. Inst. Fourier, 29 (1979), 171-200.

[4] Caracterisation et proprietés des ensembles localement pics de $A^{\infty}(D)$, Duke Math. J., 47 (1980), 763-787. 
[5] F.R. Harvey and R.O. Wells, Holomorphic approximation and hyperfunction theory on a $C^{1}$ totally real submanifold of a complex manifold, Tran. Amer. Soc., 181 (1973), 273-292.

[6] A. Iordan, Peak sets in pseudoconvex domains with isolated degeneracies, Math. Z., 188 (1985), 535-543.

[7] A. Noell, Properties of a peak sets in weakly pseudoconvex boundaries in $\mathbb{C}^{2}$, Math. Z., 186 (1984), 99-116.

[8] R.M. Range, Holomorphic functions and integral representations in several complex variables, Springer-Verlag, New York, 1986.

[9] H. Rossi, Differentiable submanifolds of complex Euclidean space, International Congress of Mathematics, Moscow, 1966.

Received November 18, 1996.

2085 Islington Avenue \#2211

ETOBICOKE, ONTARIO M9P 3R1

CANADA

E-mail address: rbelhach@acs.ryerson.ca 